\title{
W helicity, top quark spin and charge
}

\author{
Elizaveta Shabalina ${ }^{1, a}$ for the CDF, D0, ATLAS and CMS collaborations \\ ${ }^{1}$ Georg-August-Universität Göttingen, Friedrich-Hund-Platz 1, D-37077 Göttingen, Germany
}

\begin{abstract}
An overview of the recent measurements of top quark charge, spin correlations, and top quark and $\mathrm{W}$ boson polarisation in $t \bar{t}$ events is presented. The measurements were performed at the Fermilab Tevatron collider in $p \bar{p}$ collision data at $\sqrt{s}=1.96 \mathrm{TeV}$ by the CDF and D0 experiments and in $p p$ collision data at $\sqrt{s}=7 \mathrm{TeV}$ by the ATLAS and CMS experiments
\end{abstract}

\section{Introduction}

The existence of the top quark was required in the Standard Model (SM) as an isospin partner of the b-quark with its properties well defined expect for the mass. When top quark was discovered at the Fermilab Tevatron in 1995 [1] its mass appeared to be the largest of known fundamental particles and it makes this particle unique. The lifetime of the top quark, inspite being determined by the weak interactions, is at least an order of magnitude shorter than the time scale for strong interactions, implying that the top quark decays before hadronization [2] and thus its properties can be studied from the distributions of the decay products. Given precise predictions by the SM measurements of top quark properties play an important role in proving the validity of SM and can give a hint for new physics if deviations are found.

Studies of the top quark properties started at the Tevatron but were severely statistically limited until recently when the full Tevatron dataset became available. The LHC with its high rate of top pair production provides a unique opportunity to perform precision measurements of the top quark production and decay properties.

Within the SM, top quark decays to a $W$ boson and a $b$-quark with nearly $100 \%$ probability. Thus, $t \bar{t}$ events can create final states with two leptons (dilepton channel) if both $W$ bosons from top quark decay leptonically into $e v_{e}, \mu v_{\mu}$ or $\tau v_{\tau}$, single lepton $(\ell+$ jets channel) with one $W$ boson decaying leptonically and another one hadronically into $q \bar{q}^{\prime}$, or no leptons (all hadronic channel) if both $W$ bosons decay hadronically. The dilepton and single lepton channels are typically used to study top quark properties due to their clean signatures and manageable backgrounds.

The $\ell+$ jets channel is characterized by one high transverse momentum $\left(p_{T}\right)$ charged isolated lepton $(e$ or $\mu)$, large missing transverse momentum from the neutrino escaping the detector, and at least four jets, two of which originate from b-quarks. Properties measurements performed in this channel typically require at least one or two

\footnotetext{
a e-mail: Elizaveta.Shabalina@cern.ch
}

jets identified as b-jets by a b-tagging algorithm to supress the main background coming from $\mathrm{W}$ production in association with jets.

The dilepton $t \bar{t}$ signal is characterized by two high- $p_{T}$ isolated charged leptons, missing transverse momentum corresponding to the undetected neutrinos from the two leptonically decaying $\mathrm{W}$ bosons, and two b-jets. Due to the small backgrounds dominated by the $\mathrm{Z}+$ jets and diboson production this channel offers an opportunity to study top quark properties without a b-tagged jet requirement to avoid the associated systematic uncertainty.

\section{W boson helicity}

The top quark decay vertex, i.e. the Wtb vertex, is defined by the (V-A) structure of the electroweak interaction, where $\mathrm{V}$ and $\mathrm{A}$ are the vector and axial-vector contributions to the vertex. This determines the helicity states in which $\mathrm{W}$ bosons are produced in top quark decays. The next-to-next-to-leading-order (NNLO) QCD calculations predict that the fraction of longitudinal helicity $\mathrm{W}$ bosons is $F_{0}=0.687 \pm 0.005$, left-handed is $F_{L}=0.311 \pm 0.005$ and right-handed is $F_{R}=0.0017 \pm 0.0001$ [3]. These fractions can be extracted from measurements of the angular distribution of the decay products of the top quark:

$\frac{1}{\sigma} \frac{d \sigma}{d \cos \theta^{*}}=\frac{3}{4}\left(1-\cos ^{2} \theta^{*}\right) F_{0}+\frac{3}{8}\left(1-\cos \theta^{*}\right)^{2} F_{L}+\frac{3}{8}\left(1+\cos \theta^{*}\right)^{2} F_{R}$ where $\theta^{*}$ is the angle between the momentum direction of the d-type decay product of $\mathrm{W}$ (lepton, d-, or s-quark) and the reversed b-quark momentum in $\mathrm{W}$ boson rest frame, and $F_{0}+F_{L}+F_{R}=1$.

\subsection{Results from the Tevatron}

The recent measurement at the Tevatron is performed by the CDF collaboration using matrix element (ME) technique in $8.7 \mathrm{fb}^{-1}$ of data [4] in the $\ell+$ jets channel. This technique relies on calculating a likelihood for each event based on the leading order (LO) ME for $t \bar{t}$ signal which depends on the $\mathrm{W}$ helicity fractions and the dominant $\mathrm{W}+$ jets 
background. The likelihood for the selected sample is obtained as a product of per event likelihoods. This technique allows to maximize the use of kinematic event information and provides maximum sensitivity in the regime of limited statistics. A simultaneous determination of $F_{0}$ and $F_{R}$ gives: $F_{0}=0.726 \pm 0.066($ stat $) \pm 0.067$ (syst), $F_{R}=$ $-0.045 \pm 0.043$ (stat) \pm 0.058 (syst). Figure 1 shows the distributions of the reconstructed $\cos \theta^{*}$ in the $\ell+$ jets channel in the data compared to the SM prediction. All measurements of $\mathrm{W}$ boson helicity fractions from the Tevatron and LHC are consistent with the SM.

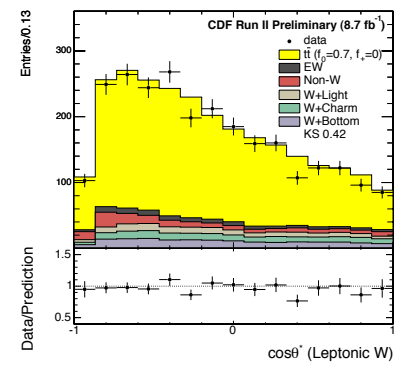

Figure 1. Distributions of the reconstructed $\cos \theta^{*}$ in the $\ell+$ jets channel in the data compared to the SM expectation [4].

\subsection{Results from the LHC}

The ATLAS collaboration performed measurements of $\mathrm{W}$ helicity fractions in the single lepton channel and dilepton channels using different methods in $1 \mathrm{fb}^{-1}$ of data [5]. To calculate $\cos \theta^{*}$ events are reconstructed in the $\ell+$ jets channel using a kinematic fitter or a $\chi^{2}$ minimization technique which both aim to find a solution most consistent with the $t \bar{t}$ production hypothesis. In the dilepton channel $t \bar{t}$ system is reconstructed by solving a set of equations and choosing the solution with the smallest product of the transverse momenta of two neutrinos.

In the template method $\mathrm{W}$ helicity fractions are extracted from the binned Poisson likelihood fit to data of the sum of background and right-handed, left-handed, and longitudinal helicity templates for the signal. The Monte Carlo generator Protos [6] was used to generate three samples with $F_{0}=1, F_{L}=1$ and $F_{R}=1$. This was achieved by choosing appropriate values for anomalous $W t b$ couplings [6]. The measured values of $F_{0}, F_{L}$ and $F_{R}$ after combining dilepton and $\ell+$ jets channels are: $F_{0}=0.66 \pm 0.06$ (stat) \pm 0.07 (syst), $F_{L}=0.33 \pm 0.03$ (stat) \pm 0.03 (syst), $F_{R}=0.01 \pm 0.03$ (stat) \pm 0.06 (syst). Figure 2 shows reconstructed $\cos \theta^{*}$ in the $\ell+$ jets channel in data overlaid with the sum of the background and $t \bar{t}$ signal using the SM and the best fit W helicity fractions. A similar method is used by the CMS collaboration to measure W helicity fractions in $2.2 \mathrm{fb}^{-1}$ of data in the $\mu+$ jets channel where different helicity configurations are created using a reweighting technique. This technique takes into account simultaneously the resolution effects and the dependence of the reconstructed $\cos \theta^{*}$ distribution on the $\mathrm{W}$ helicity fractions. The event-by-event weight that uses generated
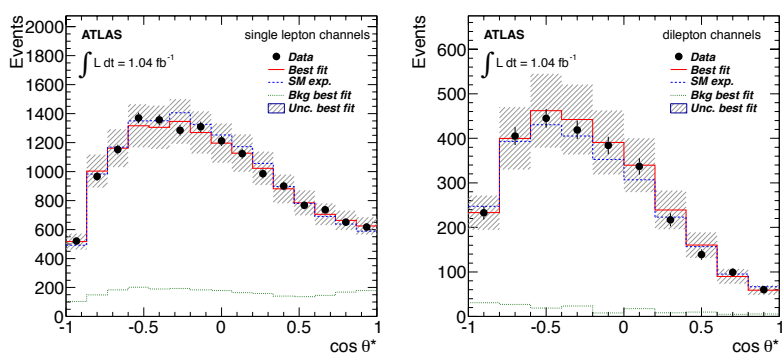

Figure 2. Distributions of the reconstructed $\cos \theta^{*}$ in the template method in the $\ell+$ jets (left) and dilepton (right) channels in the data compared to the fitted background, the SM expectation and the best fit value [5].

$\cos \theta^{*}$ value is included into the minimization procedure. The CMS measurement yields $F_{0}=0.567 \pm 0.074$ (stat) \pm 0.047 (syst), $F_{L}=0.393 \pm 0.045$ (stat) \pm 0.029 (syst),$F_{R}=$ $0.040 \pm 0.035$ (stat) \pm 0.044 (syst). Figure 3 shows $\cos \theta^{*}$ distribution in data compared to the sum of the backgrounds and SM $t \bar{t}$ signal. Information about the polarization of the

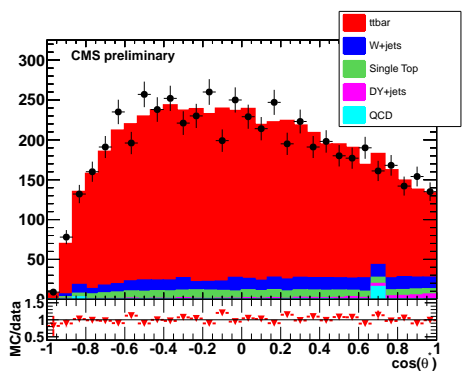

Figure 3. Distributions of the reconstructed $\cos \theta^{*}$ in the $\mu+$ jets channel in the data compared to the fitted background and the SM expectation [7].

W bosons can also be obtained using complementary observable, such as angular asymmetries $A_{+}$and $A_{-}$, defined as

$$
A_{ \pm}=\frac{N\left(\cos \theta^{*}>z\right)-N\left(\cos \theta^{*}<z\right)}{N\left(\cos \theta^{*}>z\right)+N\left(\cos \theta^{*}<z\right)} \text {. }
$$

If $z= \pm\left(1-2^{2 / 3}\right)$ for $A_{ \pm}$dependence of the latter on $F_{L}$ and $F_{R}$, respectively, cancels. The asymmetries can be related to the helicity fractions by a simple system of equations [3]. To measure the asymmetries ATLAS analysis divides $\cos \theta^{*}$ distribution into four non-uniform bins to count the number of events above and below $z$. Iterative procedure is used to correct event counts for the acceptance and resolution effects. This method was applied to dilepton and $\ell+$ jets channels. The obtained angular asymmetries are converted into $\mathrm{W}$ boson helicity fractions and the results are combined with the template method taking into account statistical and systematic correlations. The combined result is $F_{0}=0.67 \pm 0.03$ (stat) \pm 0.06 (syst), $F_{L}=$ $0.32 \pm 0.02$ (stat) \pm 0.03 (syst),$F_{R}=0.01 \pm 0.01$ (stat) \pm 0.04(syst). 


\section{Top quark charge}

In the SM top quark is a fermion with the charge of $+2 / 3$ decaying as $t \rightarrow W^{+} b$. However a number of new physics models [8] predict exotic top-like quark with the charge of $-4 / 3$. This would imply that top quark decays into $W^{-} b$, and the analysis of top quark decay products can provide an indirect measurement of its charge.

The measurement is performed in the $\ell+$ jets channel after requiring at least one identified $b$-jet and include three components. First, the charge of the leptonically decaying $\mathrm{W}$ boson based on lepton charge is determined. Then the flavor of $b$-jets is decided and, finally, the pairing of $\mathrm{W}$ bosons with $\mathrm{b}$-jets is established. Two methods are using to evaluate $b$-jet charge. The first one relies on the weighted sum of the charges of the particles associated with a b-jet. In the second one, the sign of the charge of the lepton arising from the semileptonic decay defines the sign of the b-quark.

\subsection{Results from the Tevatron}

The recent measurement of top quark charge by the CDF collaboration using $5.6 \mathrm{fb}^{-1}$ of data [9] exploits the track charge weighting algorithm to reconstruct the b-quark flavor. Figure 4 shows the distribution of the product of the $\mathrm{W}$ charge and the associated b-jet charge (combined charge) for data compared to the SM prediction. Negative (positive) values in the plot correspond to a SM like (nonSM like) pairs. The measured fraction of SM like pairs of 0.83 is translated into the $99 \%$ C.L. exclusion of the exotic model. Based on the alternative Bayesian statistical interpretation the measured value of Bayes factor allows to conclude that the data favors very strongly the SM top quark hypothesis over the exotic quark hypothesis.

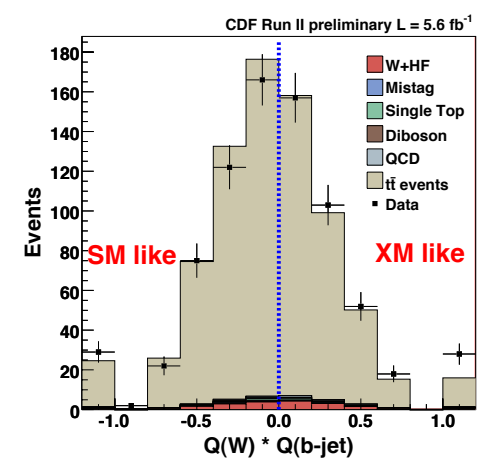

Figure 4. Distributions of the combined charge in data compared to SM expectation [9].

\subsection{Results from the LHC}

The measurement performed by the CMS collaboration in $\mu+$ jets channel is based on $4.6 \mathrm{fb}^{-1}$ of data and uses muon in jet to determine the b-quark charge [11]. Depending on the charge prediction of the top quark the events are classified into two exclusive categories corresponding to SM like and non-SM like cases. Figure 5 presents the number of events assigned to these categories compared to the SM expectation. An exotic top quark scenario predicts a flipped distribution. The hypothesis test based on this distribution allows to exclude the exotic model at $99.9 \%$ C.L.

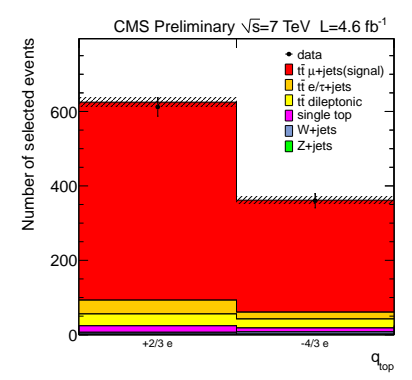

Figure 5. Distributions of the top quark charge in data compared to SM expectation [11].

The ATLAS collaboration employs both techniques to study the top quark charge in $0.7 \mathrm{fb}^{-1}$ of data. Figure 6 shows the distribution of the combined charge obtained with the track charge weighting technique for data compared to the SM and the exotic model. The mean combined charge $\left(Q_{\text {comb }}\right)$ expected for the SM top quark is marked with the red dashed line. The measured $\left\langle Q_{\text {comb }}\right\rangle$ is $-0.082 \pm 0.015$ and $-0.31 \pm 0.015$ for the track charge weighting method and the soft muon tag method to be compared to the SM prediction of $-0.082 \pm 0.013$ and $-0.234 \pm 0.011$, respectively. The measured values of $<Q_{\text {comb }}>$ for both methods are consistent with the SM and allow to exclude exotic scenario with either method at more than 5 standard deviations.

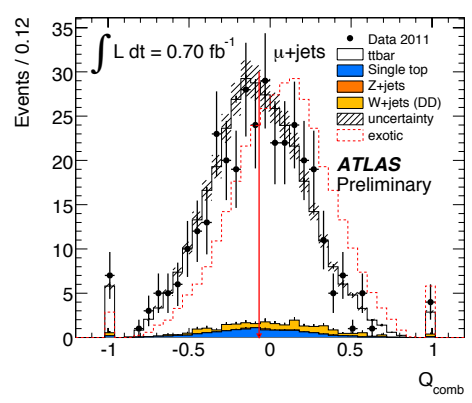

Figure 6. Distributions of the combined charge in data compared to SM expectation and the exotic top quark with the charge of $-4 / 3[10]$.

\section{Top quark spin and polarisation}

Extremely short lifetime of the top quark allows to observe the top quark spin at top quark production since hadronization effects do not deteriorate spin information which is transferred to its decay products and is reflected in their angular distributions. While the top and antitop quarks are 
produced unpolarized at the hadron colliders due to parity conservation in QCD and the unpolarised initial state, their spins are correlated [12]. The strength of the correlation can be expressed as $A=\left(N_{\uparrow \uparrow}+N_{\downarrow \downarrow}-N_{\downarrow \uparrow}-N_{\uparrow \downarrow}\right) /\left(N_{\uparrow \uparrow}+\right.$ $\left.N_{\downarrow \downarrow}+N_{\downarrow \uparrow}+N_{\uparrow \downarrow}\right)$, where $N_{\uparrow \uparrow}$ and $N_{\downarrow \downarrow}$ are the number of events with parallel spins, and $N_{\uparrow \downarrow}$ and $N_{\downarrow \uparrow}$ are the number of events with the antiparallel spins. The strength $A$ depends on the dominating production mechanism and thus is different at the Tevatron where $t \bar{t}$ pairs are produced predominantly via $q \bar{q}$ annihilation as opposed to gg fusion at the LHC. It also depends on the choice of the quantization axis, referred to as "spin basis". The optimal quantisation basis that maximises correlation strength is different at the Tevatron and the LHC and corresponds to the beam and helicity basis, respectively. For the beam basis the beam direction defines the quantization axis. For the helicity basis it is the top quark momentum direction in the $t \bar{t}$ centerof-mass frame.

First measurements of the spin correlation strength at the Tevatron were performed using double differential distribution:

$$
\frac{1}{\sigma} \frac{d \sigma}{d \cos \theta_{1} d \cos \theta_{2}}=\frac{1}{4}\left(1-C \cos \theta_{1} \cos \theta_{2}\right),
$$

where $\theta_{1}$ and $\theta_{2}$ are the angles between the direction of flight of the down-type fermions from the $\mathrm{W}$ boson decay in the respective parent $t$ or $\bar{t}$ rest frame and the chosen spin quantization axis. Coefficient $C$ is a product of the spin correlation strength at production $A$ and the amount of spin information carried by each decay product $\alpha_{i}$. Theoretical calculations at NLO QCD [13] predict that d-type decay products of $\mathrm{W}$ boson (lepton, $\mathrm{d}-$, or squark) have spin analysing power close to unity and thus the best sensitivity to the top quark spin state, while the power of b-quark is close to 0.4 . The top quark polarisation $P$ can be extracted from the distribution of the same angles $W\left(\cos \theta_{i}\right) \propto 1+\alpha_{i} P \cos \theta_{i}$. Both the spin correlation strength and polarisation are sensitive to the potential new physics contributions in top quark production and decay. Many scenarios of new physics beyond the SM predict different spin correlations or polarisation while keeping the $t \bar{t}$ production cross section within experimental and theoretical bounds [14].

\subsection{Results from the Tevatron}

The latest measurement at the Tevatron was performed by the D0 collaboration using ME technique in $5.3 \mathrm{fb}^{-1}$ of data in the dilepton and $\ell+$ jets channels [15]. The analysis calculates signal probabilities $P_{s g n}$ for each event using the LO ME for the hypothesis of correlated top quark spins $(H=c)$ as predicted by the SM, and for the hypothesis of uncorrelated spins $(H=u)$ [16]. To distinguish between correlated and uncorrelated top quark spin hypotheses, a discriminant $R$ is defined as $R=P_{s g n}(x ; H=$ $c) /\left(P_{s g n}(x ; H=u)+P_{s g n}(x ; H=c)\right)$. The fraction of events with the correlated spins $f$ is determined from the binned maximum likelihood fit to $R$ distribution in data. Combining $f$ measured in the dilepton and $\ell+$ jets channels D0 analysis obtains $f=0.85 \pm 0.29$ (stat + syst). This measurement provided first evidence of SM spin correlation at 3.1 standard deviations.

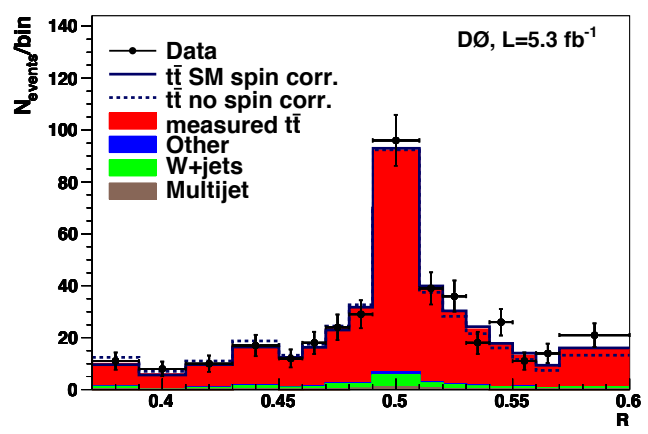

Figure 7. The distribution of the discriminant $R$ for the $\ell+$ jets channel of the D0 analysis [15]. The expectation for spin correlation as predicted by the SM $(f=1)$ and the case of no spin correlation $(f=0)$, as well as the $t \bar{t}$ contribution for the measured value of $f$ are shown.

\subsection{Results from the LHC}

At the LHC the dominating gg fusion mechanism of the $t \bar{t}$ production allows to study spin correlations in the dilepton channel using the azimuthal angle $\Delta \phi$ between the two charged leptons from $\mathrm{W}$ boson decay in the laboratory frame [17]. This angle is sensitive to the interference between like-helicity gluon pairs which dominate top pait production at low $t \bar{t}$ invariant mass. This method is very advantageous experimentally because the angles are well measured in the detector and reconstruction of the top quarks is not required.

The ATLAS collaboration performed a measurement of spin correlation strength using $2.1 \mathrm{fb}^{-1}$ of data. The spin correlation is extracted by comparing $\Delta \phi$ distribution in data to the $\Delta \phi$ distributions from the SM $t \bar{t}$ signal simulation and the one with uncorrelated top spins. The fit determines the fraction of events in data consistent with the SM prediction $f$. Negative values of $f$ correspond to an anticorrelation of the top and antitop quark spins. A value of $f=0$ implies that the spins are uncorrelated and values of $f>0$ indicate a larger strength of the $t \bar{t}$ spin correlation than predicted by the SM. The combined measurement in $e e, e \mu$ and $\mu \mu$ channels yields $f=1.3 \pm 0.14$ (stat) ${ }_{-0.22}^{+0.27}$ (syst). This can be translated into the measurement of spin correlation $A$ using the SM theoretical prediction $A_{S M}$. For the helicity basis $A_{S M}$ is 0.31 at NLO [13] yielding $A_{\text {helicity }}=0.40 \pm 0.04(\text { stat })_{-0.07}^{+0.08}$ (syst). The data are inconsistent with the hypothesis of zero spin correlation with a significance of 5.1 standard deviations. Figure 8 shows the reconstructed $\Delta \phi$ distribution for the sum of the three dilepton channels in data overlaid with the SM and uncorrelated $t \bar{t}$ MC samples and with the expected backgrounds.

The CMS collaboration performed the measurement using the same technique with $5.0 \mathrm{fb}^{-1}$ of data [19] and 


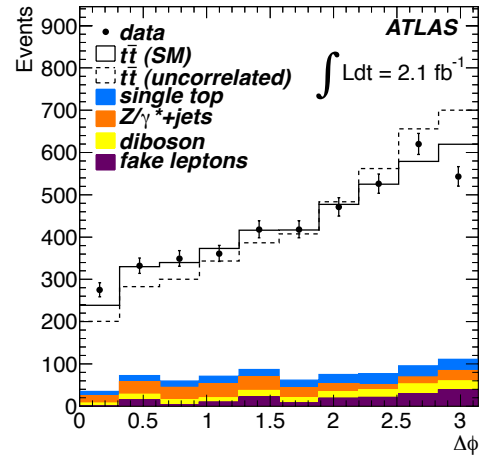

Figure 8. The reconstructed charged lepton $\Delta \phi$ distribution in data in the dilepton channel for the ATLAS measurement [18] compared to the sum of the expected backgrounds and SM or uncorrelated $t \bar{t}$ MC samples. Both the SM and the uncorrelated $t \bar{t}$ contributions are normalized to the fitted number of $t \bar{t}$ events.

obtained $f=0.74 \pm 0.08$ (stat) \pm 0.24 (syst) and $A_{\text {helicity }}=$ $0.24 \pm 0.02$ (stat) \pm 0.08 (syst). The spin correlations were investigated furthermore by CMS through other variables and for different $t \bar{t}$ invariant masses. At higher invariant masses, $t \bar{t}$ production through unlike-helicity gluons, producing $t \bar{t}$ pairs in anti-aligned orientations $(\downarrow \uparrow$ or $\uparrow \downarrow)$, identical to configurations dominating $t \bar{t}$ production at the Tevatron through $q \bar{q}$ annihilation, becomes dominant. The following asymmetry variables are defined:

$$
A_{\Delta \phi}=\frac{N\left(\Delta \phi_{l^{+} l^{-}}<\pi / 2\right)-N\left(\Delta \phi_{l^{+} l_{-}}>\pi / 2\right)}{N\left(\Delta \phi_{l^{+} l^{-}}<\pi / 2\right)+N\left(\Delta \phi_{l^{+} l-}>\pi / 2\right)}
$$

and

$$
A_{c 1 c 2}=\frac{N\left(\cos \theta_{l^{+}} \times \cos \theta_{l^{-}}>0\right)-N\left(\cos \theta_{l^{+}} \times \cos \theta_{l^{-}}<0\right)}{N\left(\cos \theta_{l^{+}} \times \cos \theta_{l^{-}}>0\right)+N\left(\cos \theta_{l^{+}} \times \cos \theta_{l^{-}}<0\right)}
$$

where $\cos \theta_{l^{+}}\left(\cos \theta_{l^{-}}\right)$is the cosine of the production angle of the lepton with respect to the direction of the parent top or anti-top in the $t \bar{t}$ rest frame. Asymmetry $A_{c 1 c 2}$ provides a direct measurement of the spin correlation and unlike $A_{\Delta \phi}$ requires event reconstruction. The study of both variables in different $t \bar{t}$ invariant mass regions can be useful to discriminate between the different models which could explain the large deviation of the observed $t \bar{t}$ forward-backward asymmetry at the Tevatron from the SM prediction. Table 1 summarises measured and predicted by simulation inclusive asymmetries and asymmetries in the high mass region of $t \bar{t}$ pair $M_{t \bar{t}}>450 \mathrm{GeV}$ and shows no significant deviation between the data and simulation. The reconstructed $\Delta \phi$ and $\cos \theta_{l^{+}} \times \cos \theta_{l^{-}}$distributions are corrected for the limited acceptance and detector resolution effects to determine asymmetries at the parton level. The background-subtracted and unfolded differential cross sections for $\Delta \phi$ and $\cos \theta_{l^{+}} \times \cos \theta_{l^{-}}$are shown in figure 9 compared to POWHEG simulation [20] and the NLO calculation [21]. The unfolded asymmetries are $A_{\Delta \phi}=-0.097 \pm 0.015$ (stat) \pm 0.036 (syst) and $A_{c 1 c 2}=-0.015 \pm 0.037$ (stat) \pm 0.055 (syst) in agreement with the values in simulation of $-0.119 \pm 0.0004$ and $-0.063 \pm 0.0004$, respectively.

\begin{tabular}{l|cc}
\hline Asymmetries & Data & Simulation \\
\hline$A_{\Delta \phi}$, inclusive & $-0.158 \pm 0.010$ & $-0.171 \pm 0.002$ \\
$A_{c 1 c 2}$, inclusive & $-0.062 \pm 0.011$ & $-0.087 \pm 0.002$ \\
\hline$A_{\Delta \phi}, M_{t \bar{t}}>450 \mathrm{GeV}$ & $-0.378 \pm 0.019$ & $-0.384 \pm 0.003$ \\
$A_{c 1 c 2}, M_{t \bar{t}}>450 \mathrm{GeV}$ & $-0.019 \pm 0.016$ & $-0.044 \pm 0.003$ \\
\hline
\end{tabular}

Table 1. Reconstructed and simulated inclusive asymmetries and in the region of $M_{t \bar{t}}>450 \mathrm{GeV}$ obtained by the CMS analysis [19]. Uncertainties are statistical only.
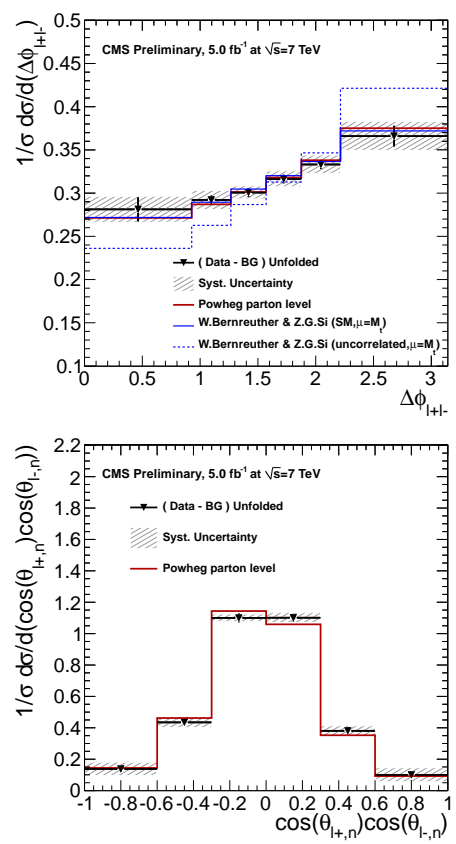

Figure 9. The background-subtracted and unfolded differential cross sections for $\Delta \phi$ and $\cos \theta_{l^{+}} \times \cos \theta_{l^{-}}$[19].

The CMS collaboration applied unfolding technique to measure top quark polarisation in the dilepton events with $5.0 \mathrm{fb}^{-1}$ of data [22]. Figure 10 shows the backgroundsubtracted and unfolded distribution of $\cos \theta_{l^{+}}$with the parton level prediction from POWHEG. Polarisation $P$ obtained from the difference in the number of events with positive and negative $\cos \theta_{l^{+}}$is $-0.009 \pm 0.029$ (stat) \pm 0.041 (syst), consistent with the SM prediction of unpolarised $t \bar{t}$ production.

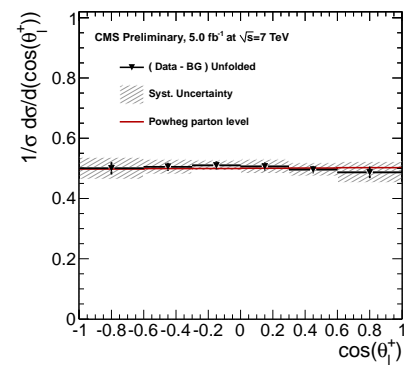

Figure 10. The background-subtracted and unfolded distribution of $\cos \theta_{l^{+}}$[22]. 
The ATLAS analysis [23] uses $\ell+$ jets events and a template fit to the reconstructed $\cos \theta_{l}$ distribution in data to extract polarisation. The templates are created for fully positively and negatively polarised top quarks by reweighting the simulated $t \bar{t}$ events such that the generated distributions of $\cos \theta_{l}$ are equal to $0.5\left(1+\cos \theta_{l}\right)$ and $0.5\left(1-\cos \theta_{l}\right)$. The fit obtains the fraction of positively polarised top quarks $f=0.470 \pm 0.009$ (stat) ${ }_{-0.032}^{+0.023}$ (syst) in agreement with the SM value of $f=0.5$. This is equivalent to $P=-0.060 \pm 0.018$ (stat) $)_{-0.064}^{+0.046}$ (syst). Figure 11 shows the reconstructed $\cos \theta_{l}$ distribution in the data overlaid with the best fit result as well as the signal distributions for fully positive and negative polarisations and no polarisation in the $\mu+$ jets channel.

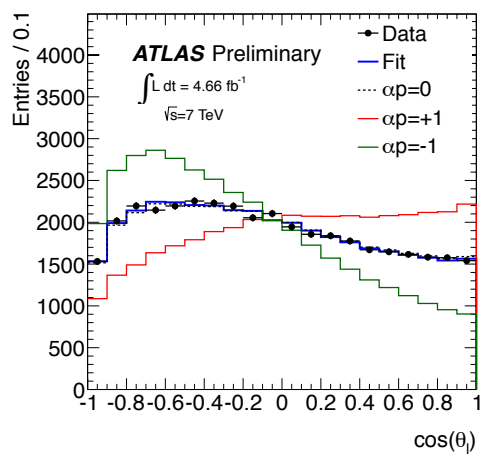

Figure 11. Reconstructed $\cos \theta_{l}$ distribution in data overlaid with the best fit result and the signal distributions for fully positive, negative polarisations and zero polarisation in the $\mu+$ jets channel [23].

\section{Conclusions}

Large sample of top quarks collected at the LHC provides unique opportunities for precise studies of top quark properties initiated at the Tevatron. With the dataset collected in 2011 at the $\sqrt{s}=7 \mathrm{TeV}$ spin correlations in $t \bar{t}$ production were observed at the LHC, the first measurements of the top polarisation were performed and the precise measurements of $\mathrm{W}$ boson helicity allowed to set stringent limits on the anomalous Wtb couplings. Following the Tevatron, exotic top quark charge has been excluded. Up to now all measurements of top quark properties carried out at the LHC are in agreement with the SM.

The precision of the majority of the results discussed in this document is limited by the systematic uncertainties. The most important are the jet energy scale calibration, $t \bar{t}$ signal model and the knowledge of top quark mass. Therefore further improvement of the precision will crucially depend on the improved understanding of the detector effects as well as of various aspects of the $t \bar{t}$ signal model.

\section{References}

[1] CDF Collaboration, F. Abe et al., Phys. Rev. Lett. 74, 2626 (1995); D0 Collaboration, S. Abachi et al., Phys. Rev. Lett. 74, 2632 (1995).

[2] I. Bigi, Y. Dokshitzer, V. Khoze, J. Kühn, and P. Zerwas, Phys. Lett. B 181, 157 (1986).

[3] A. Czarnecki, J.G. Korner and J.H. Piclum, Phys. Rev. D 81, 111503 (2010).

[4] CDF collaboration, CDF note 10855 (2012).

[5] ATLAS collaboration, JHEP 1206, 88 (2012).

[6] J. Aguilar-Saavedra, J. Carvalho, N.F. Castro, F. Veloso and A. Onofre, Eur. Phys. J. C50, 519 (2007).

[7] CMS collaboration, CMS-PAS-TOP-11-020 (2012).

[8] D. Chang, We-Fu Chang, E. Ma, Phys. Rev. D59, 091503 (1999).

[9] CDF collaboration, CDF note 10460 (2011).

[10] ATLAS collaboration, ATLAS-CONF-2011-141 (2011).

[11] CMS collaboration, CMS-PAS-TOP-11-031 (2012).

[12] G. Mahlon and S. Parke, Phys. Rev. D 53, 4886 (1996); T. Stelzer and S. Willenbrock, Phys. Lett. B 374, 169 (1996), G. Mahlon and S. Parke, Phys. Lett. B 411, 173 (1997).

[13] A. Brandenburg, Z. G. Si and P. Uwer, Phys. Lett. B539, 235 (2002); W. Bernreuther, A. Brandenburg, Z. G. Si, and P. Uwer, Phys. Rev. Lett. 87, 242002 (2001).

[14] D. Krohn, T. Liu, J. Shelton, and L.-T. Wang, Phys. Rev. D84 074034 (2011); R. Frederix and F. Maltoni, JHEP 01, 047 (2009); M. Arai, N. Okada, and K. Smolek, Phys. Rev. D79, 074019 (2009) 074019; G. L. Kane, G. A. Ladinsky, and C. P. Yuan, Phys. Rev. D45, 124 (1992); K. Cheung, Phys. Rev. D55, 4430 (1997).

[15] D0 collaboration, Phys. Rev. Lett. 108, 032004 (2012).

[16] K. Melnikov and M. Schulze, Phys. Lett. B 700, 17 (2011).

[17] G. Mahlon and S. Parke, Phys. Rev. D 81, 074024 (2010).

[18] ATLAS collaboration, Phys. Rev. Lett. 108, 212001 (2012).

[19] CMS collaboration, CMS-PAS-TOP-12-004 (2012).

[20] P. Nason, J. High Energy Phys. 11040 (2004); S. Frixione, P. Nason, and C. Oleari, JHEP 11 (2007) 070; S. Alioli, P. Nason, C. Oleari, and E. Re, JHEP 06043 (2010).

[21] W. Bernreuther and Z. G. Si, Nucl. Phys. B 837, 90 (2010).

[22] CMS collaboration, CMS-PAS-TOP-12-016 (2012).

[23] ATLAS collaboration, ATLAS-CONF-2012-133 (2011). 